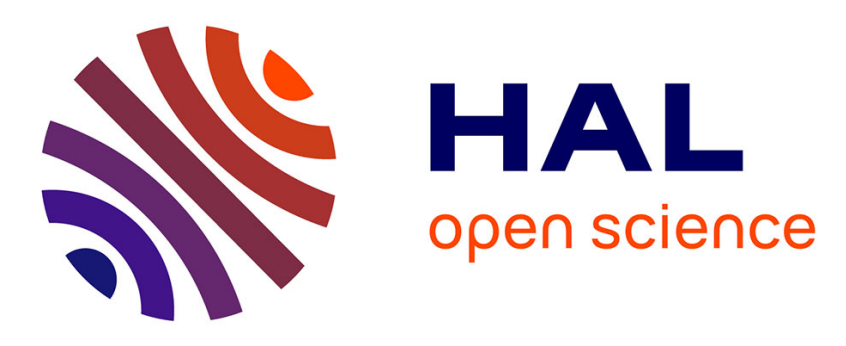

\title{
Meta-models Combination for Reusing Verification Techniques
}

Hui Zhao, Ludovic Apvrille, Frédéric Mallet

\section{To cite this version:}

Hui Zhao, Ludovic Apvrille, Frédéric Mallet. Meta-models Combination for Reusing Verification Techniques. MODELSWARD 2019 - 7th International Conference on Model-Driven Engineering and Software Development, Feb 2019, Prague, Czech Republic. pp.39-50, 10.5220/0007261000390050 . hal-02080768

\section{HAL Id: hal-02080768 https://hal.inria.fr/hal-02080768}

Submitted on 28 May 2019

HAL is a multi-disciplinary open access archive for the deposit and dissemination of scientific research documents, whether they are published or not. The documents may come from teaching and research institutions in France or abroad, or from public or private research centers.
L'archive ouverte pluridisciplinaire HAL, est destinée au dépôt et à la diffusion de documents scientifiques de niveau recherche, publiés ou non, émanant des établissements d'enseignement et de recherche français ou étrangers, des laboratoires publics ou privés. 


\title{
Meta-models Combination for Reusing Verification Techniques*
}

\author{
Hui Zhao \\ Université Côte d'Azur, CNRS, Inria, I3S \\ Ludovic Apvrille \\ LTCI, Telecom ParisTech, Université Paris Saclay \\ Frédéric Mallet \\ Université Côte d'Azur, CNRS, Inria, I3S
}

\begin{abstract}
The design of Cyber-Physical systems (CPS) demands to combine discrete models of pieces of software (cyber) components with continuous models of physical components. Such heterogeneous systems rely on numerous domains with competencies and expertise that go far beyond traditional software engineering: systems engineering. In this paper, we explore a model-based approach to systems engineering that advocates the composition of several heterogeneous artifacts (called views) into a sound and consistent system model. Rather than trying to build the universal language able to capture all aspects of systems, we rather propose to bring together small subsets of languages to focus on specific analysis capabilities while keeping a global consistency of all these small pieces of languages. We take as an example, an industrial process based on Capella, which provides (among others) a large support for functional analysis from the requirements to the deployment of components. Even though, Capella is already quite expressive, it does not provide a direct support for schedulability analysis. However, AADL is an language also dedicated to system analysis. It focuses on schedulability analysis, but that does not provide direct support for functional analysis. Rather than trying to extend either Capella or AADL into always more expressive languages to add the missing features we rather extract a pertinent subset of both languages to build a view adequate for conducting schedulability analysis of Capella functional models. Our language is generic enough to extract pertinent subsets of languages and combine them to build views for different experts. It also maintains a global consistency between the different views.
\end{abstract}

\section{Introduction}

Developers of Cyber-Physical Systems (CPSs) have to deal with different domains, each of them having different characteristics. It is seldom the case that one development platform or a single language can adapt to all aspects with assumption one-sizefits-all. Therefore developers have to rely on domain-specific languages to handle the different domains problem. This results not only in a proliferation of languages but also

\footnotetext{
${ }^{*}$ This work has been supported by the French government, through the UCA ${ }^{\mathrm{JEDI}}$ Investments in the Future project managed by the National Research Agency (ANR) with the reference number ANR-15-IDEX-0001.
} 
increases the design complexity of CPS. Meanwhile, the gaps between languages and platforms brought additional problems such as coherency and consistency problems, which are exposed at integration and simulation stages. This also further exacerbates the complexity, make the complexity skyrocketing.

To tackle these problems, engineers need an approach which can efficiently combine already existing languages. The goal is not only to combine the different languages seamlessly but also to benefit from the advantages of each language. Two solutions are possible. (i) A first solution is to continuously integrate the necessary languages into an existing development platform, thus leading to progressively building a comprehensive development platform. However, this could lead to a never ending process resulting in a gigantic framework, which would be difficult to use and maintain. (ii) A second solution is to keep each language (or tool) isolated, and relate some of the elements from each language (sub) meta-model in order to conduct the different analyses offered by each approach (e.g., scheduling analysis, safety analysis). In this second solution, each domain expert can work independently. Yet, since each language has its own characteristics such as syntax and semantics, we have to eliminate the gaps between them and handle the consistency issues. Therefore, our contribution is to propose a formal combining approach to link two modeling languages. To do so, we define how to relate two (sub-)metamodels. Then, for two given models $m_{1}, m_{2}$ conforming each of the two languages, $m_{2}$ can be augmented with some of the information of $m_{1}$ so as to perform verification on enriched models (e.g., scheduling, timing, safety), and then to trace the verification results back to $m_{1}$

In this paper, we selected SysML and AADL to demonstrate the relevance of our approach. These languages are supported by the tools Capella/Arcadia and OSATE2 ${ }^{1}$, respectively. The paper is organized as follows. We present our contribution with first our workflow and then with the formal definition of a set of combination rules and operators. Then, in section 3, we apply these operators on functional and physical views. In section 4, train traction controlling systems are used to demonstrate architecture and scheduling analysis. Section 5 presents the related work. Finally, section ?? concludes the paper and gives our future work.

Some special notes for this paper are: 1) When we mention Arcadia, it means SysML-based methodology, the language is SysML. 2) In sections 2, 3 and 4, all elements on the left of transformation rules belong to metamodels of Arcadia and all elements on the right are from the AADL metamodel. The two metamodels have been imported by default, and we thus omit the prefix (e.g., MM.Arcadia.function) for conciseness.

\section{Approach}

\subsection{Workflow}

The workflow of our approach is shown as figure 1. On the one hand the Arcadia methodology and Capella modeling platform focus on several high-level phases of engineering and system functional analysis. On the other hand, the AADL focuses on structural modeling to describe the concrete execution behaviors of components.Hence, in this paper, we aim to enrich the Arcadia model with elements of the AADL model for which a relationship to Arcadia has been established. Firstly, we proposed a set of operators to specify the relationships at the M2 level, which contains corresponding

\footnotetext{
${ }^{1}$ http://osate.org/index.html
} 


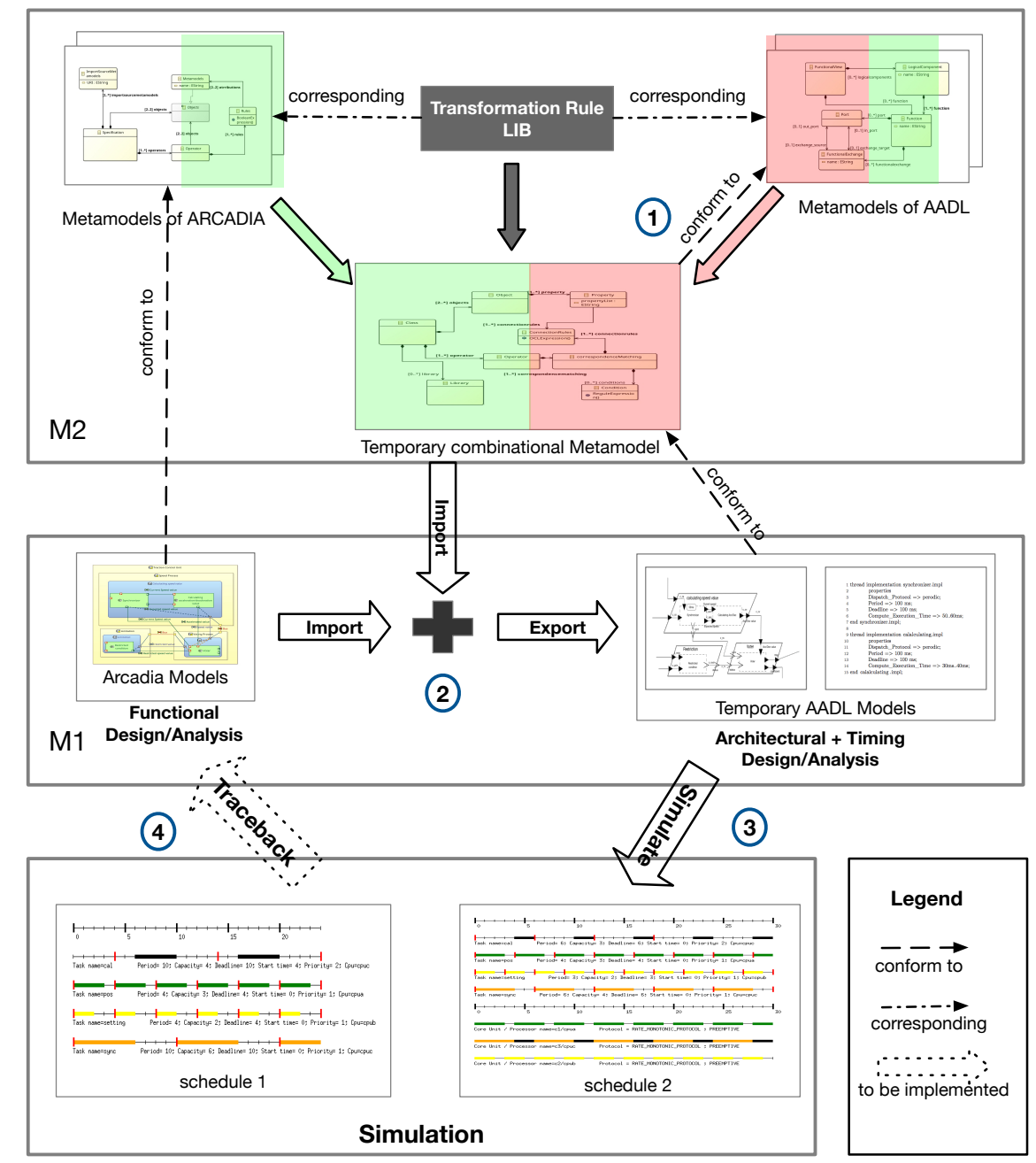

Figure 1: Overview of Workflow

relationships between Arcadia and AADL metamodels and the relationship of other additional attributes. Those relations serve to constitute the transformation system, and the set of all relationships is called Transformation Rule Library (TRL).

In this case, Arcadia and AADL have similar parts but different names (e.g., function in Arcadia and thread in AADL, shown as green part in the figure). To capture and analyze the new features which not exist in Arcadia, Some additional attributes must be added (e.g., period and execution time, shown as red part). Once the metamodels are prepared, we can get a temporary combinational metamodel (TCM) by using TRL at step 1. Moreover, one can use TCM and an existing Arcadia model to export to an AADL model adding new attributes manually at step 2 . The new AADl concrete models will be exported into OSATE for further editing. Next, it will be used to perform the scheduling simulation in an analysis tool Cheddar at step 3 . The design flaws, conflicts of time and resource would be detected. These results help designers to improve original models at step 4 and fix vulnerabilities or improve the system performances. We expect to be able to automatically trace back to the original model in the near future. 


\subsection{Operators and Rule expression}

According to the workflow in the above subsection, initially, we have a set of metamodels for Arcadia and AADL. We propose a set of operators to manipulate the metamodels, then using these operators to represent operations between metamodels (e.g., transforming, creating, ignoring) in a systematic way. We also provided semantic definitions of operators as well as corresponding writing rules, to help the integration engineer custom-tailor metamodel as they needed. In the following parts of the paper, in order to simply and clearly describe the transformation rules, several operators and their semantics are defined. (see the table 1).

\begin{tabular}{lr}
\hline Symbol of operator & Meaning \\
\hline$\Gamma$ & Transformation Rule \\
$;$ & End of rule \\
$\rightsquigarrow \rightarrow$ & Transfer \\
$<>$ & Parent node \\
\{\} & Attribute \\
{[]} & Optional element \\
$\mid$ & Separation of elements \\
\{\}$+$ & Attribute is to be created \\
$\neg$ & Ignore \\
\hline
\end{tabular}

Table 1: Symbols of transformation rule expression

\subsubsection{Structure of rule}

One rule begins with " $\Gamma$ " and end with ";". What we have to note is each rule can contain one source object which in the left of transfer symbol $\rightsquigarrow$ and one or more objects in the right side are target objects. The parent node is enclosed within the angle brackets " $<>$ " (if it has one parent node). it means each rule should write as below:

$$
\Gamma<\text { parent }>\text { source } \rightsquigarrow \text { target; }
$$

Each part of the object separated by ":". The attribute group is enclosed with parentheses " \{\}$" ;$ square braces "[ ]" delimit optional elements and the alternatives are separated by a pipe "|". For example, The port has a directional attribute called Direction which could be in or out. Shown as below:

$$
P_{\text {ort }}:\{\text { Direction }[\text { in } \mid \text { out }]\}
$$

\subsubsection{Creating operator}

In the case of creating a new attribute, put the name of an attribute in the parentheses with plus " \{\}$+$ ", is used to present the option is to be created. An example as below, an attribute type of component port will be created with three optional values (data, event, data and event):

$$
\text { Port : }\{\text { Type }[\text { data|event } \mid \text { dataevent }]\}+
$$




\subsubsection{Ignoring operator}

For some ignored attributes and objects are denoted with " $\neg$ " which is in front of the ignored object.

An example of transformation rule expressions is in listing 1. In particular, the number of attributes of the target object may be greater than the number of attributes of the source object in the case of a new object created.

$1 \Gamma P_{\text {ort }}:\{$ Direction[in|out] $\left.\}\right) \leadsto<$ feature $>:$ Port: $\{$ Direction[in|out] $\}:\{$ Type[data|event $\mid$ data event $\left.]\right\}+$;

$2 \Gamma P P \leadsto<$ feature $>$ :Port: $\{$ Direction[in|out] $\}+:\{$ Type[data|event|data event $]\}+$;

$3 \Gamma P_{\text {ort }}: \neg\{$ ordering $\left.\}\right)$;

$4 \Gamma E x_{\text {fun }}:\{$ Source $\}:\{$ Target $\} \rightsquigarrow<$ connections $>$ :connection: $\{$ source $\}:\{$ target $\}$;

Listing 1: An example of transformation rules

\subsection{Arcadia2AADL model transformation}

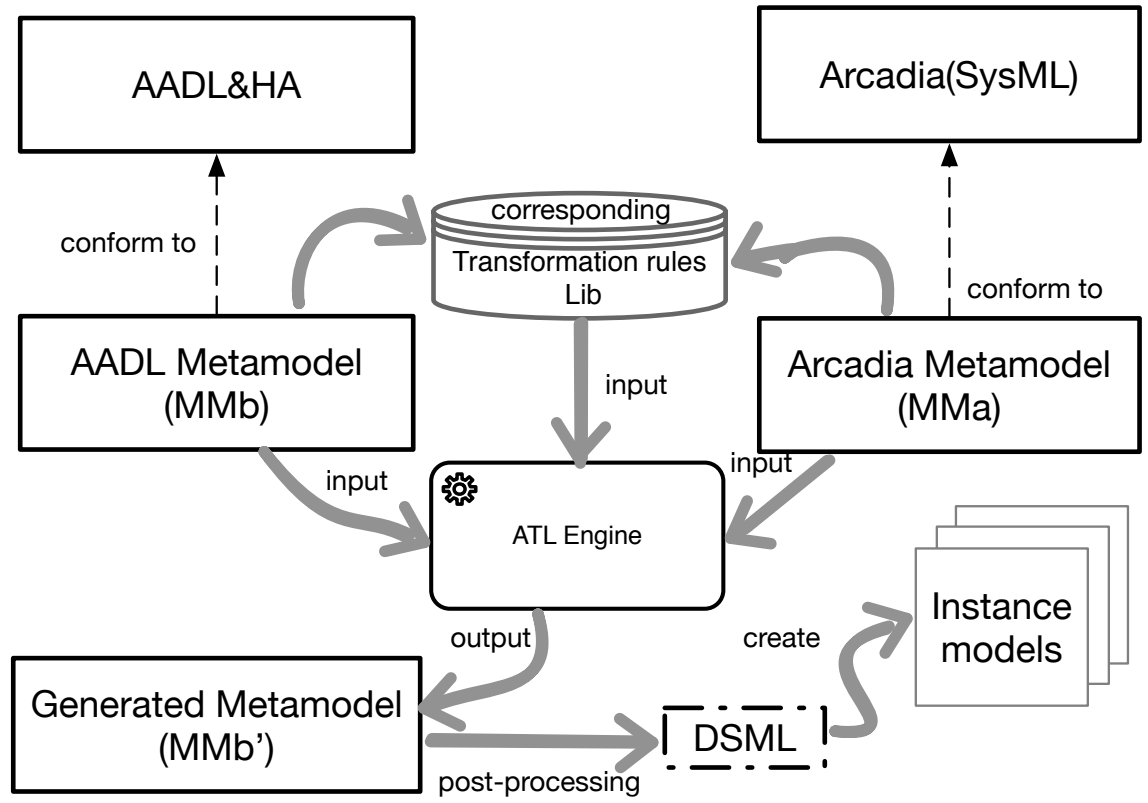

Figure 2: Structure of Arcadia2AADL model transformation

Based on the defined transformation rules, Arcadia2AADL transformation can create a temporary metamodel according to original metamodels and TRL. ATL serves as a transformation engine. For more detailed information about ATL, the reader is referred to [Jouault et al., 2006][Jouault et al., 2008]. The Structure of Arcadia2AADL model transformation is shown in Fig 2. AADL metamodels (denoted as MMb) and Arcadia metamodels (denoted as MMa) are imported (input) into ATL engine, and the engine is going to read transformation rules library and then the transformation engine exports (output) a temporary metamodel (denoted as metamodel MMb') which is a union subset of AADL and Arcadia and conform to AADL metamodel. The generated metamodel is then further post-processed as a DSML (domain-specific modeling language) in ecore format which can be used to create instance models. Therefore, all 
instance models are conform to this metamodel and will be used for further simulation and analysis.

\section{Transformation Rule Library}

As we described in the above section, the Transformation Rule Library (TRL) play an important role in the transformation process. Hence, in this section, we present how to construct a TRL from the following three views, functional view and physical view. Each view contains the metamodels which are from the subset of AADL and Arcadia.

\subsection{Functional view}

\subsubsection{Logical components in Arcadia}

Logical components in Arcadia contain a set of member elements, such as logical component containers, functions, ports, and functional exchanges. In the Arcadia, Functional diagrams consist of a set of SysML blocks and its interactions, named Logical components; The notion of Logical component enables better expression of system engineering semantics compared to SysML, and particularly, reduces the bias towards software. SysML block definition diagrams (BDDs) and internal block diagrams (IBDs) are assigned to different abstract and refined layers, respectively. The definition of a block in SysML can be further detailed by specifying its parts; ports, specifying its interaction points; and connectors, specifying the connections among its parts and ports. This information can also be visualized using logical components in Arcadia. Definition 3.1.1 shows a metamodel of logical components.

Definition 3.1.1. (Logical Component) A logical component $(\boldsymbol{L C})$ is 5-tuple,

$$
\mathcal{L} C=<C_{\text {omp }}, F_{\text {un }}, P_{\text {ort }}, E x_{\text {fun }}, M_{c f}>
$$

where, $C_{\text {omp }}=\left\{\bigcup_{F_{u n}}\right\}$ is a logical component container which contains a set of functional elements.

$F_{u n}$ is a finite set of functional block include their name and id attributes. $P_{\text {ort }}$ is a finite set of functional ports including directions and allocation attributes. $E x_{f u n} \subseteq$ $P_{\text {ort }} \times P_{\text {ort }}$ denotes a finite set of functional exchange (connection) between two functional ports, it must be pair, one is source, another is target. $M_{c f}: \Sigma F_{u n} \rightarrow C_{o m p}$ allocate functions to a logical component container.

In Figure 3, there is a functional instance model of a part of a vehicle traction control unit in ARCADIA as an example. The blue rectangle is named logical component in Arcadia, but we consider it as a function's container, we thus call it logical component container $C_{o m p}$ in this paper. The green rectangle are functions $F_{u n}$ which are contained by $C_{o m p}$. The element $M_{c f}$ has represented this allocation relationship between logical component containers and functions $M_{c f}: \Sigma F_{u n} \rightarrow C_{o m p}$. The deep green square with the white triangle is the outgoing port $\left(P_{\text {ort }}\right)$, which connects to an incoming port $\left(P_{\text {ort }}\right)$ that is drawn as a red square with white triangle and the green line is the functional exchange between two functional ports $\left(E x_{f u n}\right)$. 


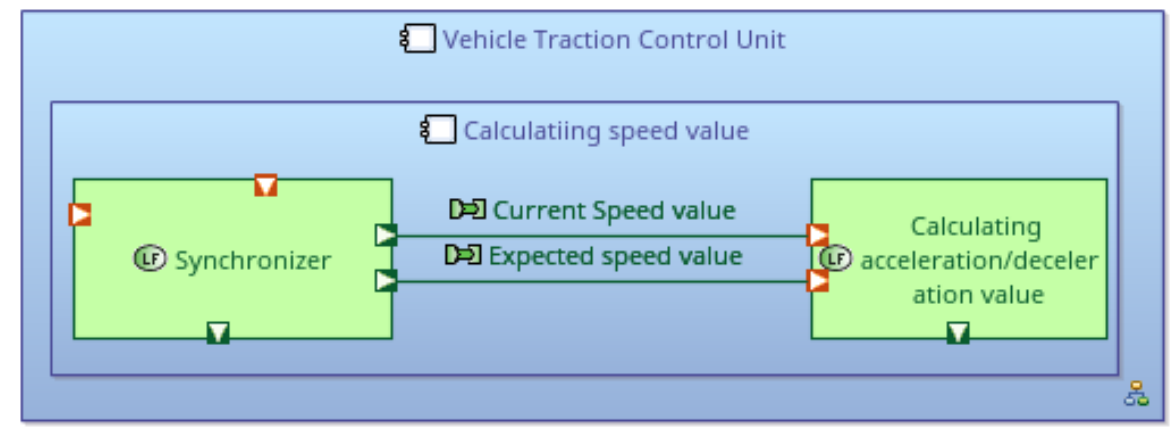

Figure 3: An example of functional view of vehicle traction control unit in ARCADIA

\subsubsection{The subset of software in AADL}

AADL is able to model a real-time system as a hierarchy of software components, predefined software component types in the category of the components such as thread, thread group, process, data, and subprogram are used to model the software architecture of the system.

Definition 3.1.2. (Software Composition) A SC is a 4-tuple:

$$
\mathcal{S C}=<\text { Type, Port, Connection, Annex }>
$$

where Type specifies the type of components (e.g, system, process, thread). Port is a set of communication point of component. Port could be different types such as data port, event port and data event port. And, port can specify the direction such as in port, out port, in out port. Connection is used to connect ports in the direction of data/control flow in uni- or bi-directional.

\subsubsection{Functional elements transformation rules}

Table 2 shows the correspondence between AADL and Arcade elements. The Additional attributes column are the attributes to be crated during the transformation. According to this table, we can easily write the transformation rules to transforming Arcadia to AADL on functional parts, denoted $\mathcal{L} C \rightsquigarrow \mathcal{S C}+\mathcal{H} \mathcal{A}$. See an example in listing 2 .

$1 \Gamma C_{\text {omp }} \rightsquigarrow$ Type[ system|process]:\{Runtime_Protection[true $\mid$ false] $\}+$;

$2 \Gamma F_{\text {un }} \rightsquigarrow$ Type $[$ abstract $\mid$ thread $]:\left\{\right.$ Dispatch $\_$Protocol[Periodic|Aperiodic|Sporadic|Background|Timed $\mid$ Hybrid]\}+; 3

Listing 2: Functional elements transformation rules example

\subsection{Physical view}

\subsubsection{Execution Platform in AADL}

Processor, memory, device, and bus components are the execution platform components for modeling the hardware part of the system. Ports and port connections are 


\begin{tabular}{|c|c|c|}
\hline Arcadia & AADL & Additional attributes \\
\hline LC container $\left(C_{o m p}\right)$ & System,Process & $\{$ Runtime_Protection[true|false] $\}+$ \\
\hline Function $\left(F_{u n}\right)$ & Abstract, Thread & $\{$ Dispatch_Protocol[Periodic|Aperiodic \\
\hline & & Sporadic|Background|Timed|Hybrid] $\}+$ \\
\hline Port $\left(P_{\text {ort }}\right)$ & Port & $\{$ Type[data|event|data event $]\}+$ \\
\hline Functional Exchange $\left(E x_{f u n}\right)$ & Connection & $\bigcirc$ \\
\hline$\bigcirc$ & Annex & Type[abstract| thread]: $\{$ annex $\}+$ \\
\hline Physical Node $\left(N_{\text {ode }}\right)$ & Device,Memory,Processor,Bus & $\begin{array}{l}\{\text { Dispatch_Protocol }\}+:\{\text { Period }\}: \\
\{\text { Deadline }\}+:\{\text { priority }\}+\end{array}$ \\
\hline Physical Port $(P P)$ & $\bigcirc$ & $\neg P P$ \\
\hline Physical Link $(P L)$ & Bus/BusAccess & $\begin{array}{l}{[\{\text { Allowed_Connnection_Type }\}+:} \\
\{\text { Allowed_Message_Size }\}+ \\
\mid\{\text { Allowed_Physical_Access }\}+ \\
:\{\text { Transmission_Time }\}+]\end{array}$ \\
\hline
\end{tabular}

Table 2: Functional and Physical elements correspondence table

provided to model the exchange of data and event among components. Functional and non-functional properties like scheduling protocol and execution time of the thread can be specified in components and their interactions.

Definition 3.2.1. (Execution Platform) A EP component is defined as a six tuples:

$$
\mathcal{E P}=<E C, B A, C_{\text {onn }}>
$$

where, EC defines the execution component such as processor, memory, bus and device. BA defines the BusAccess which is interactive approach between bus component and other execution platform components. $C_{\text {onn }} \subseteq E C \times E C$ denotes a finite set of connection between two components via bus device.

\subsubsection{Physical components in Arcadia}

The physical component in Arcadia consists of physical Node, Port and Link. The Physical Port and Link correspond to port and bus connection in AADL. There are some choices when the physical Node is translated to AADL such as device, memory, and processor, hence the designer has to point out what type of target component during transformation by using transformation rule express.

Definition 3.2.2. (Physical Components) A Physical components is 3 tuples,

$$
P C=<N_{\text {ode }}, P P, P L>
$$

where, $N_{\text {ode }}$ is a execution platform, named node in Arcadia, it could be different type of physical component (e.g, processor, board). PP is the physical component port. PL is physical link, it could be assigned a concrete type such as bus.

Figure 4 is a physical instance model of a part of a vehicle traction control unit in ARCADIA. We can see the yellow parts are the physical node $\left(N_{\text {ode }}\right)$ and the red line is the physical link $(P L)$ named bus in this case which connects to two physical ports $(P P)$, the small square in dark yellow. 


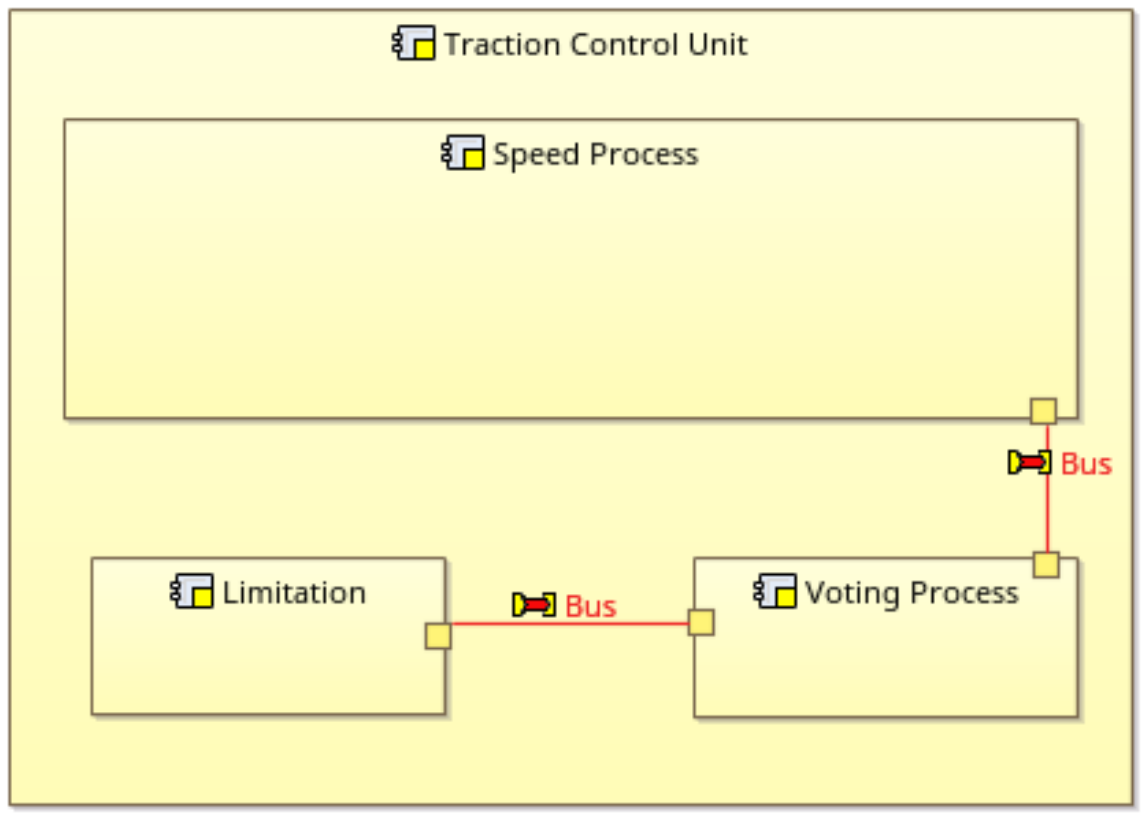

Figure 4: An example of physical view of vehicle traction control unit in ARCADIA

\subsubsection{Physical elements transformation rules}

According to Table 2, we can easily write the transformation rules for physical elements. Listing 3 shows a part of the code to transform the physical component from Arcadia to AADL.

$1 \Gamma N_{\text {ode }} \rightsquigarrow[$ Device $\mid$ Process $\mid$ Memory $\mid$ Bus $]:\{$ Dispatch_Protocol $\}+:\{$ Period $\}:\{$ Deadline $\}+:\{$ priority $\}+$; $2 \Gamma P P \leadsto \neg P P$;

$3 \Gamma P L \rightsquigarrow$ Bus/BusAccess:[\{Allowed_Connnection_Type $\}+:\{$ Allowed_Message_Size $\}+\mid\{$ Allowed_Physical_Access $\}+:\{$ Transmission_Time $\}+]$;

Listing 3: Physcial elements transformation rules example

Let us focus on the physical link part (see line 3). The Bus device could be a logical resource or hardware component. Hence, the bus device has different properties depending on the role. When the bus is considered as a logical resource, it contains the properties Allowed_connection_type and Allowed_Message_Size. When the bus is hardware, it contains Allowed_Physical_Access and Transmission_Time. Therefore, we write the rules that either

$\{$ Allowed_Connnection_Type $\}+:\{$ Allowed_Message_Size $\}+$

or

$\{$ Allowed_Physical_Access $\}+:\{$ Transmission_Time $\}+$ 


\section{Case Study}

To show the efficacy of our approach in transforming and using produced AADL models to analyze the properties, this section presents the experimental results of analyzing the traction controlling unit of railway signaling system. By using our proposed approach, we transfer and extend Arcadia metamodel, and design AADL using OSATE2 with the generated metamodel. once the concrete models have been created, the scheduling property is chosen to show analysis ability through Cheddar tool.

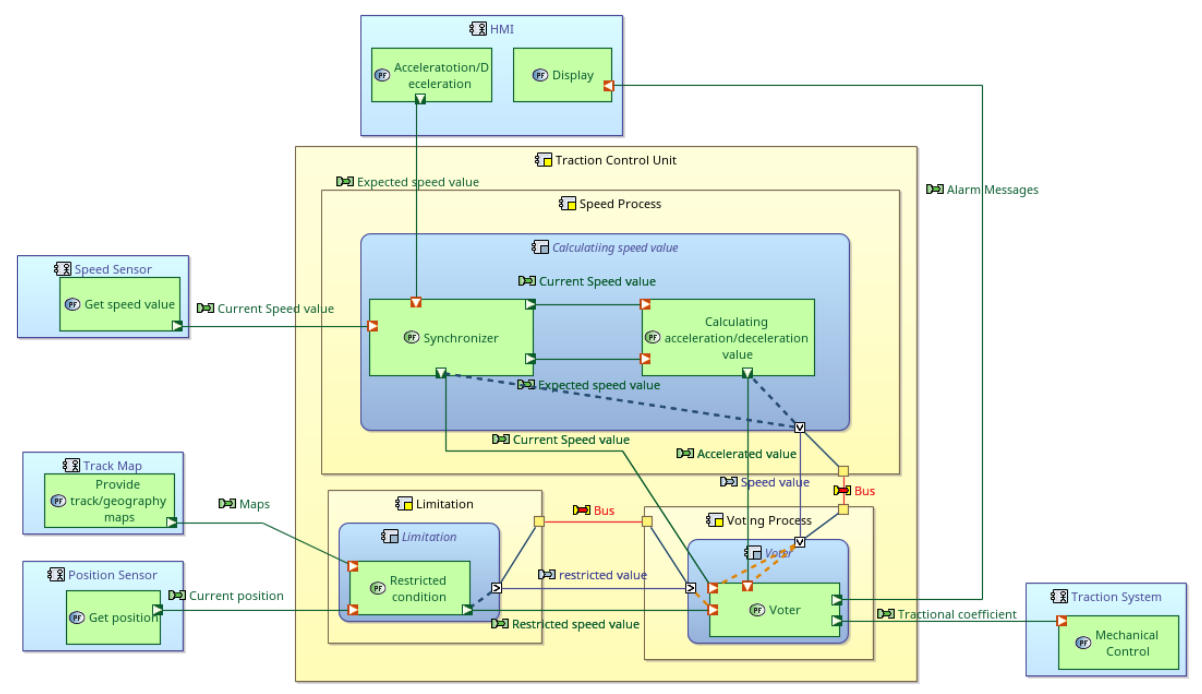

Figure 5: Arcadia model of TCU system

\subsection{Train traction controlling system (TCU)}

Train movement is the calculation of the speed and distance profiles when a train is traveling from one point to another according to the limitations imposed by the signaling system and traction equipment characteristics. As the train has to follow the track, the movement is also under the constraints of track geometry, and speed restrictions and the calculation becomes position-dependent. The subsystem of calculating the traction effective and speed restrictions is therefore critical to achieving train safe running. Nowadays, Communication based train control (CBTC) is the main method of rail transit (both urban and high-speed train) which adopts wireless local area networks as the bidirectional train-ground communication [Zhu et al., 2009]. To increase the capacity of rail transit lines, many information-based and digital components have been applied for networking, automation and system inter-connection, including general communication technologies, sensor networks, and safety-critical embedded control system.

This paper uses a subsystem which called traction controlling system (TCU) from signaling system of high-speed railways, we use it to illustrate the model transformation from engineering level to detailed architectural level and verified the instance models. The functional modules such as calculation and synchronization will be transformed using our approach, and then non-functional properties such as timing correctness and resource correctness will be verified by schedule analysis tool Ched- 


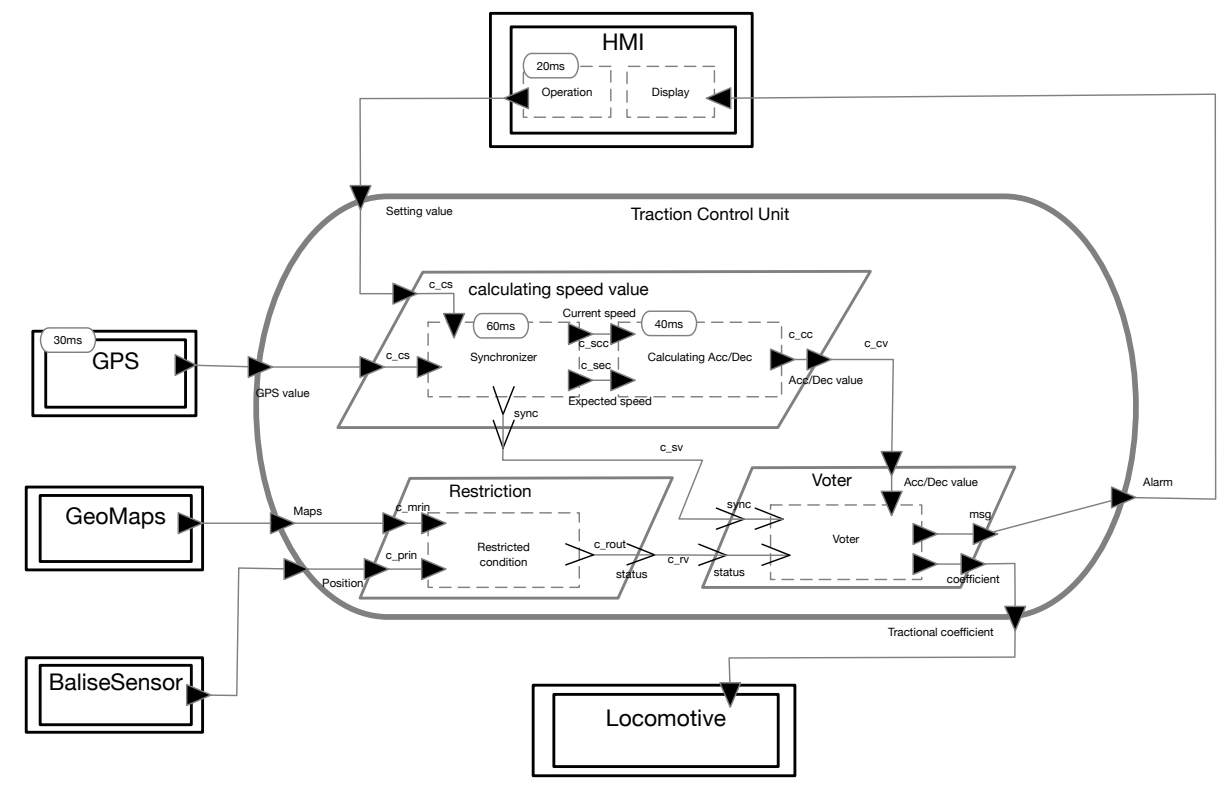

Figure 6: AADL model of TCU system

dar [Singhoff et al., 2004].

First, we start with component functional views and physical view analysis by designing system models in Arcadia (shown in figure 5). The functions of the traction control system are to collect the external data by sensors such as a speed sensor. The data from Balise sensors is used to determinate the track block, and then it is going to seek the speed restriction conditions by matching accurate positioning (if the track blocks are divided fine enough) and digital geometric maps data. Meanwhile, calculating speed unit received the speed data from GPS and speed control commands from HMI (Human-Machine Interface) periodically. GPS data provides speed value periodically, and HMI data send the operation command (e.g., expected speed value), then the calculating unit has to output an acceleration value and export to the locomotive mechanical system. Although they are periodic, the external data do not always arrive on time due to transmission delay or jitter. Therefore, we should use a synchronizer to make sure they are synchronized. Otherwise, the result would be wrong with asynchronous data. Similarly, to ensure the correctness of the command of acceleration (or deceleration), we applied a voting mechanism which can ensure the result is correct as much as possible. The voter must have the synchronized signal and restriction condition to dedicate to output the acceleration coefficient request to the locomotive system. The AADL diagram shown as figure 6.

\subsection{Model transformation}

Using the Arcadia2AADL tool, the metamodel of the TCU system in Capella is translated into the corresponding AADL metamodel with the rules and approach which describes in section 3. For instance, on the one hand, the function class is translated into the thread in AADL. To analyze the timing properties, several attributes also have been added such as protocol type, deadline, execution time, period.

On the other hand, the physical part element Node translates to the processor in this 


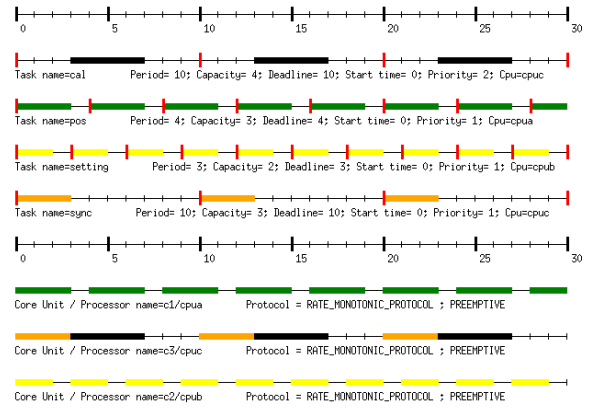

(a) Schedule 1 with idle time

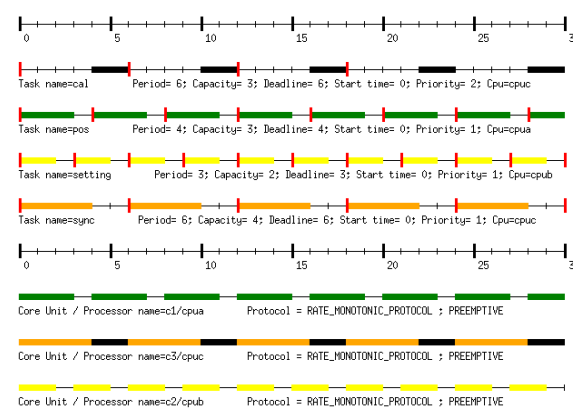

(b) Schedule 2 with compact time

Figure 7: Simulation results of task schedule

case. Differ from simple physical Node in Arcadia; the processor element attaches rich properties such as scheduling protocol (scheduler type), process execution time. The allocation relationships on both physical and functional parts are translated into AADL as well.

\subsection{Schedule verification}

The external data and internal process work sequentially is an essential safety requirement of the system, and each task should be scheduled properly. However, in real-world, the risk of communication quality and rationality of scheduling must be taken into account. Therefore, the schedule verification is a way to evaluate system timing property. An Ada framework called Cheddar which provides tools to check if a real-time application meets its temporal constraints. The framework is based on the real-time scheduling theory and is mostly written for educational purposes [Marcé et al., 2005].

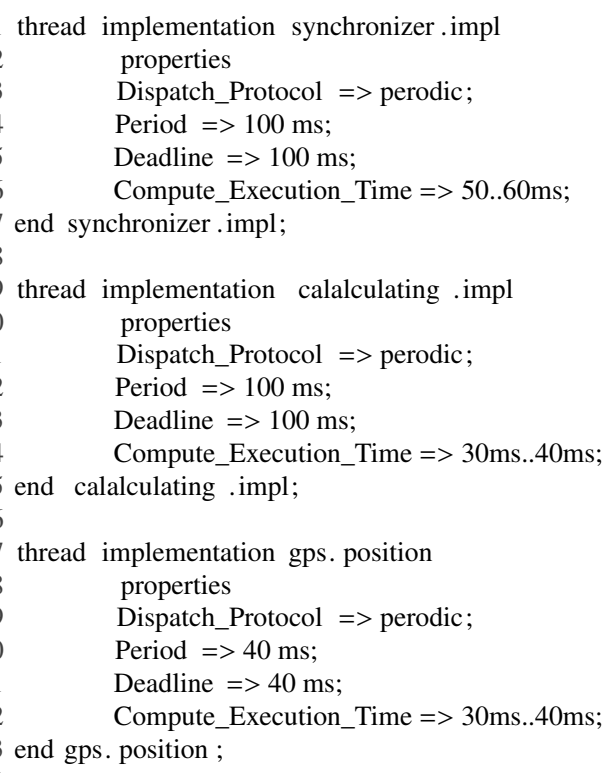




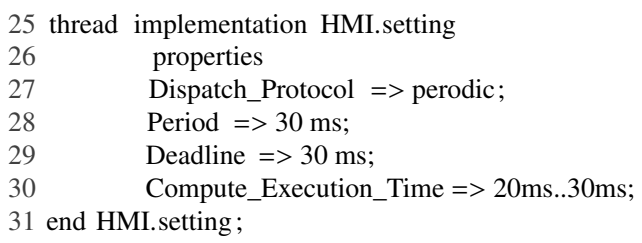

Listing 4: Setting of scheduling properties

Listing 4 shows a set of 4 periodic tasks (cal, pos, sync and setting) of TCU respectively defined by the periods 100,100, 40 and 30, the capacities 60, 40, 30 and 20, and the deadlines 100, 100, 40 and 30. These tasks are scheduled with a preemptive Rate Monotonic scheduler (the task with the lowest period is the task with the highest priority).

For a given task set, if a scheduling simulation displayed XML results in the Cheddar. One can find the concurrency cases or idle periods (see left of figure 7, comprise the software part and physical device part). People change the parameters directly and reload simulation; a feasible solution can be applied instead. After tuning, finally, the appropriate setting has displayed as in right of figure 7. According to this simulation result, people can correct the properties value in AADL, thereby ensure the correctness of system behavior timing properties.

\section{Related work}

A considerable number of studies have been proposed on extending UML-like profile to AADL and model transformation methods. This section provides a brief introduction to these works.

An approach for translating UML/MARTE detailed design into AADL design has proposed by Brun et al. [Brun et al., 2008]. Their work focuses on the transformation of the thread execution and communication semantics and does not cover the transformation of the embedded system component, such as device parts. Similarly, in [Turki et al., 2010], Turki et al. proposed a methodology for mapping MARTE model elements to AADL component. They focus on the issues related to modeling architecture, and the syntactic differences between AADL and MARTE are well handled by the transformation rules provided by ATL tool, yet they did not consider issues related to the mapping of MARTE properties to AADL property. In [Ouni et al., 2016], Ouni et al. presented an approach for transformation of Capella to AADL models target to cover the various levels of abstraction, they take into account the system behavior and the hardware/software mapping. However, the formal definition and rigorous syntactic of transformation rules are missed.

The scientists have proposed some specific methods to weave the models as well as metamodels formally such as [Jezequel, 2008], Degueule has proposed Melange, a language dedicated to merging languages [Degueule et al., 2015], and similar works like [Ramos et al., 2007]. However, the structural properties are not supported.

Behjati et al. describe how they combined SysML and AADL in [Behjati et al., 2011] and provided a common modeling language (in the form of the ExSAM profile) for specifying embedded systems at different abstraction levels. De Saqui-Sannes et al. [De Saqui-Sannes and Hugues, 2012] presented an MBE with TTool and AADL at the software level and demonstrated with flight management system. Both of their works do not provide the description in a formal way. 
Compared with current studies, the approach proposed in this paper has the following features:

1. Arcadia is chosen as the transformation source. Arcadia provides a broad view of system engineering as well as refined functional and physical views.

2. A proper subset of AADL has been chosen as the transformation target including functional software composition, execution platform. We use it to describe continuous behaviors of Cyber-Physical System.

3. All of the transformations is considered at metamodel level, and then a generated synthesized metamodel can be used to create concrete AADL models for further analysis.

4. Transformation rules are formally defined, and then it is readable by human and easier to verify the correctness of transformation.

\section{Conclusions and future work}

This paper describes a collaborative design approach between system engineering methodology Arcadia (based on SysML) and architectural design language AADL using transformation at metamodel level. We first present our approach and implementation procedures using ATL. Then, we give a formal description of the key modeling elements of Arcadia and AADL, respectively. Then translation rules from these Arcadia metamodels to AADL are formally defined. Finally, a case study of train traction controlling system is used to demonstrate the transformation from engineering concerned design into an architectural refinement design which can be further analyzed by scheduling properties. In our future work, we will study the translation rules for more elements of Arcadia and also for comprehensive SysML elements, even for others UML-like profiles such as MARTE. At the same time, we will continue to explore the AADL and its annex to support more analysis and formal verification of system design. Besides, the safety-critical systems have become a trend in industrial files. We will study the extension of AADL with verification of safety properties with transformation methodology. For further discussions on this topic, please refer to full text [Zhao et al., 2019].

\section{References}

[Behjati et al., 2011] Behjati, R., Yue, T., Nejati, S., Briand, L., and Selic, B. (2011). Extending SysML with AADL concepts for comprehensive system architecture modeling. In European Conference on Modelling Foundations and Applications, pages 236-252. Springer.

[Brun et al., 2008] Brun, M., Vergnaud, T., Faugere, M., and Delatour, J. (2008). From UML to AADL: an Explicit Execution Semantics Modelling with MARTE. In ERTS 2008.

[De Saqui-Sannes and Hugues, 2012] De Saqui-Sannes, P. and Hugues, J. (2012). Combining SysML and AADL for the design, validation and implementation of critical systems. In ERTS2 2012, page 117.

[Degueule et al., 2015] Degueule, T., Combemale, B., Blouin, A., Barais, O., and Jezequel, J.M. (2015). Melange: A meta-language for modular and reusable development of dsls. In Proceedings of the 2015 ACM SIGPLAN International Conference on Software Language Engineering, pages 25-36. ACM, ACM.

[Jezequel, 2008] Jezequel, J.-M. (2008). Model driven design and aspect weaving. Software and Systems Modeling, 7(2):209-218. 
[Jouault et al., 2008] Jouault, F., Allilaire, F., Bézivin, J., and Kurtev, I. (2008). ATL: A model transformation tool. Science of Computer Programming, 72(1-2):31-39.

[Jouault et al., 2006] Jouault, F., Allilaire, F., Bézivin, J., Kurtev, I., and Valduriez, P. (2006). ATL: a QVT-like transformation language. a QVT-like transformation language. ACM, New York, USA.

[Marcé et al., 2005] Marcé, L., Singhoff, F., Legrand, J., and Nana, L. (2005). Scheduling and Memory Requirements Analysis with AADL. In Proceedings of the 2005 Annual ACM SIGAda International Conference on Ada, pages 1-10, New York, NY, USA. ACM.

[Ouni et al., 2016] Ouni, B., Gaufillet, P., Jenn, E., and Hugues, J. (2016). Model Driven Engineering with Capella and AADL. page 0 .

[Ramos et al., 2007] Ramos, R., Barais, O., and Jezequel, J.-M. (2007). Matching modelsnippets. In International Conference on Model Driven Engineering Languages and Systems, pages 121-135. Springer.

[Singhoff et al., 2004] Singhoff, F., Legrand, J., Nana, L., and Marcé, L. (2004). Cheddar - a flexible real time scheduling framework. SIGAda, pages 1-8.

[Turki et al., 2010] Turki, S., Senn, E., and Blouin, D. (2010). Mapping the MARTE UML profile to AADL. In Proceedings of the 3rd International Workshop on Model Based Architecting and Construction of Embedded Systems (ACES-MB 2010), pages 11-20. Citeseer.

[Zhao et al., 2019] Zhao, H., Apvrille, L., and Mallet., F. (2019). Meta-models combination for reusing verification techniques. In Proceedings of the 7th International Conference on Model-Driven Engineering and Software Development - Volume 1: MODELSWARD,, pages 39-50. INSTICC, SciTePress.

[Zhu et al., 2009] Zhu, L., Zhang, Y., Ning, B., and Jiang, H. (2009). Train-ground communication in CBTC based on 802.11 b: Design and performance research. In Communications and Mobile Computing, 2009. CMC'09. WRI International Conference on, pages 368-372. IEEE. 